\title{
Holistic vs. attribute repetition effects in classifying stimuli
}

\author{
G. R. LOCKHEAD, PAUL GRUENEWALD, and MICHAEL KING \\ Duke University, Durham, North Carolina 27706
}

\begin{abstract}
The effect of the just previous stimulus on the current response was determined in three speeded classification experiments. When the stimuli were perceptual patterns, and when they were words, there was marked facilitation if the previous stimulus was identical to the current stimulus. When successive perceptual stimuli were composed of identical elements, but in a changed configuration, there was no measureable sequential effect on "same" response times. These results are for integral stimuli. When stimuli are not integral, as with some studies in the literature, it is suggested that each two-dimensional nonintegral stimulus is treated by the observer as two objects. In those cases, responses can be facilitated by repetition of that object on which responses were based on the previous trial, and this can occur when a value of the other dimensions (other objects) has not repeated. Accordingly, the processing of integral and nonintegral stimuli must be considered separately when attempting to evaluate process models. Further, facilitation due to stimulus repetitions does not account for all of the differences in response times between various classification tasks. These additional differences may be attributed to a multidimensional range effect analogous to the range effects found in psychophysical tasks.
\end{abstract}

Commonly used information processing tasks require observers to classify stimuli according to one or more predefined dimensions. These tasks frequently use only two dimensions ( $X$ and $Y$ ) with two levels on each dimension. This defines four possible stimuli: X1Y1, $\mathrm{X} 1 \mathrm{Y} 2$, X2Y1, X2Y2. Different classification tasks use either different combinations of two stimuli or they use all four stimuli. There are usually many trials in which one of the task stimuli is randomly selected and presented to an observer, who classifies the stimulus according to some rule. Three rules are typical: (1) Two stimuli are used and the observer is required to sort along one dimension while the other dimension is held constant (i.e., X1Y1 vs. X2Y1); (2) two stimuli are used and the observer discriminates between covarying two-dimensional stimuli (X1Y1 vs. X2Y2, or X2Y1 vs. X1Y2); or (3) all four stimuli are used and sorting is along one dimension while the other dimension varies in an unrelated manner (X1Y1 and $X 1 Y 2$ vs. $X 2 Y 1$ and $\mathrm{X} 2 \mathrm{Y} 2$, or $\mathrm{X} 1 \mathrm{Y} 1$ and $\mathrm{X} 2 \mathrm{Y} 1$ vs. $\mathrm{X} 1 \mathrm{Y} 2$ and $\mathrm{X} 2 \mathrm{Y} 2$ ). These are referred to, respectively, as univariate, correlated, and or thogonal tasks.

It has frequently been reported that classification performance on orthogonal tasks, in which any of four possible integral stimuli occur on a trial, is slower and less accurate than is performance on correlated and univariate tasks, in which only two stimuli are used (cf. Garner, 1974). It is easier to classify stimuli along dimension $\mathrm{X}$ when the level of $\mathrm{Y}$ is held constant from trial to trial (univariate task) than when the level of the irrelevant dimension varies randomly (orthogonal task). The orthogonal task has been designated as a gating task (Posner, 1964) or a filtering task (Fitts \& Biederman,
1965) because the varying irrelevant dimension has been considered to contribute information that must be gated or filtered out before the proper classification can be made and "the integral nature of the stimulus dimensions makes it very difficult to filter or ignore these changes" (Taylor, 1977, p. 479).

An alternative suggestion to account for the slow performance in orthogonal tasks has been offered by Felfoldy (1974). He had observers classify rectangles that varied in height and width according to univariate, correlated, and orthogonal sorting procedures. Felfoldy found that sequentially repeated items were responded to most quickly in all tasks. He suggests that the relatively poor performance in orthogonal tasks occurs because stimuli repeat less often on successive trials in the four-stimulus condition (orthogonal tasks) than in the two-stimulus conditions (univariate and correlated tasks). Only $25 \%$ of the orthogonal trials are repetitions, while $50 \%$ of both the univariate and correlated trials are stimulus repetitions. Since a stimulus is identified more readily when it is repeated on successive trials (cf. Bertelson, 1961, 1965, and many subsequent reports), the reduction of stimulus repetitions is potentially able to account for "interference" in the orthogonal task. It is not that there is interference from variations in the irrelevant dimension; rather, there is less facilitation because there are fewer repetitions of the same stimulus.

A model that states that the observer processes the entire integral stimulus before he can analyze and categorize it in terms of a specified attribute is consistent with results from classification studies using integral stimuli. By that holistic notion, the ability to 
identify a stimulus object depends on discriminability, that is, on the distance of that stimulus from other potential stimuli in psychological space (Lockhead, 1972). Additionally, the locus of attention in that space affects the ease of identification (Hutchinson \& Lockhead, 1977). If a stimulus occurs where attention is currently focused (as with stimulus repetition, priming, or expectancy), that stimulus is more readily identified than if it occurs at some other locus. When it is necessary for attention to shift, as when an unexpected or not readily distinguishable event occurs, additional time is required (Posner \& Snyder, 1975; Gruenewald \& Lockhead, Note 1). In classification studies like those discussed here, in which there is no priming, it may be appropriate to assume that attention is often at the locus of that stimulus presented on the previous trial. In that case, attention shifting predicts that performance is better in the univariate and correlated tasks than in the orthogonal tasks, because the former tasks have more repetitions and thus require fewer occurrences of attention shifting. Additionally, because stimuli are generally more removed from each other in similarity space in the correlated task than in the univariate task (Lockhead \& King, 1977), the discriminability aspect of this processing concept is consistent with the finding that correlated tasks are generally easier than are univariate tasks.

The studies in this report were designed to extend examination of the sequential effects in orthogonal tasks, as investigated by Felfoldy (1974), and to provide a further test of the holistic model. Felfoldy reported that repeating both attributes of a stimulus facilitates responses. The purpose here is to determine if the relatively poor performance associated with orthogonal classification tasks is due to less frequent repetitions of attributes (levels on a dimension or features), or if the poorer performance is, rather, due to less frequent repetitions of the object. That is, is the performance decrement attributable to fewer repetitions of common dimension levels, of attributes, or is it because the identical stimuli, the same loci in similarity space, repeat less often? One way to separate the two possibilities is to use more than one configuration constructed of the same attributes in each cell in the bivariate classification. This is done for perceptual stimuli in Experiment 1 .

\section{EXPERIMENT 1}

Stimuli were constructed such that there were two sets of forms with identical attributes but with different configurations. If classification tasks involve initially processing the whole pattern and then deciding the value of the sought attribute, repeating the same configuration should produce fast response times, but repetition of the same attributes arranged in a different configuration should not. Alternatively, if it is the attributes that are initially processed, as in systems like Selfridge's (1959) pandemonium model, then repeating the same attributes or features on successive trials should also facilitate response times, and this should be independent of configuration.

\section{Method}

Stimuli. The stimuli were pairs of horizontal and vertical lines that were either 11 or $14 \mathrm{~mm}$ long. The lines were arranged to form rectangles or number signs. The resulting eight stimuli, four stimuli of each configuration, are shown in Figure 1.

Subjects. Four paid students served as subjects. Each was run individually.

Procedure. The observer sat in front of a $16 \times 22 \mathrm{~cm}$ oscilloscope in a darkened sound-attenuating room. A small joystick was moved left by the observer if the presented stimulus was narrow (any stimulus in the left column of Figure 1) and was moved right if the stimulus was wide. The instructions were to sort the stimuli as quickly as possible while attempting to make as few errors as possible. When ready, the observer moved the joystick in either direction to begin the study; the joystick automatically returned to the central position. A randomly selected stimulus appeared in the center of the screen and remained there until the observer responded, at which time the screen erased. The next stimulus appeared about $520 \mathrm{msec}$ after the response. There were two sessions each of 50 practice trials followed by two blocks of 440 data trials. The first session

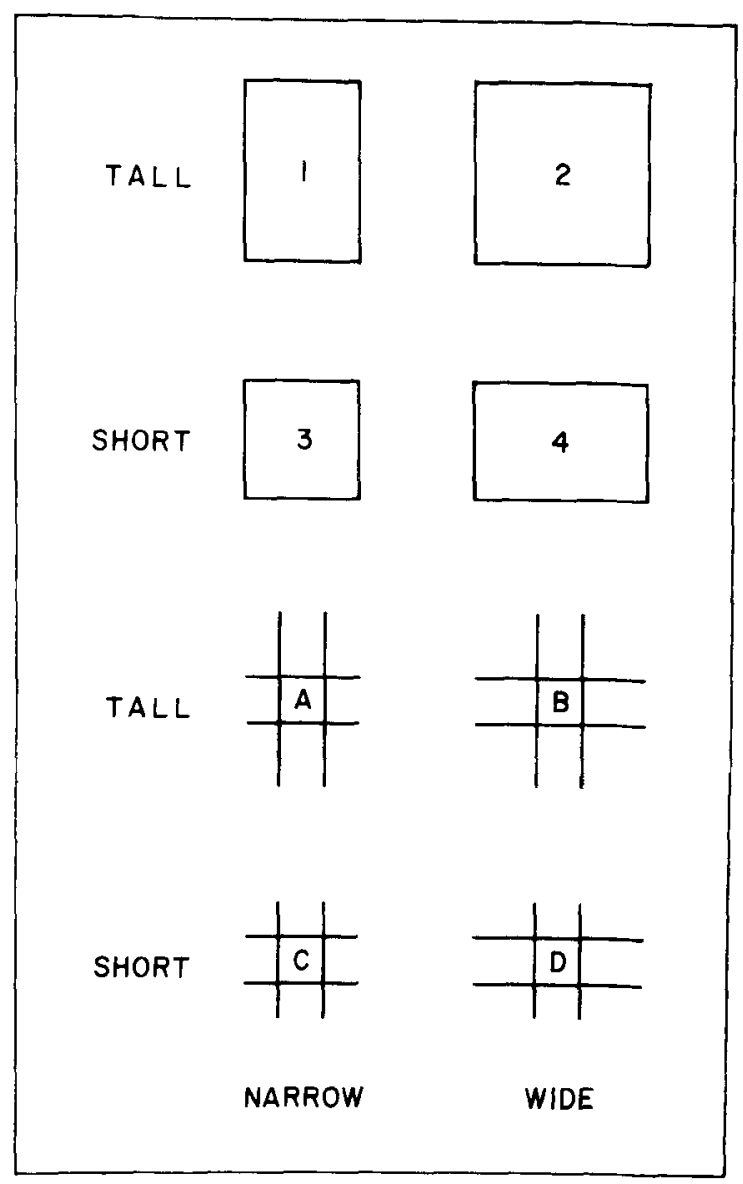

Figure 1. The stimuli used in Experiment 1. The labels have been added for identification. 
was considered as practice and only the data from the second session are reported here.

\section{Results}

The average error -rate across the four subjects was $5.2 \%$ of the responses. Error trials and trials following an error were not considered in the sequential effects analysis.

The question asked is whether the time to classify a stimulus according to a particular dimension level depends on repetition of the immediately previous stimulus or on the immediately previous dimension level. The average response times in the different conditions were calculated for each observer. The averages of those times appear in Table 1 , which also shows the sequences that constitute the observations in each cell. For example, referring both to Figure 1 and to Table 1 , on those trials that the same response was repeated (wide or narrow) and the same configuration was repeated (rectangle or number sign), but the level of the irrelevant dimension switched (tall to short or short to tall), the relevant temporally adjacent stimuli contributing to the $507-\mathrm{msec}$ average time were $1-3$, $24, A-C$, and B-D, including both sequential orders of these stimuli.

A sequential conditions by practice by subjects analysis of variance was performed on the data, producing the eight cells in Table 1 . The differences between sequential conditions were significant $[\mathrm{F}(7,21)=5.78, \mathrm{p}<.001]$. According to a NewmanKeuls test $(\alpha=.01)$, response times were faster when the identical stimulus repeated on successive trials than in all other conditions. No other conditions $(\alpha=.05)$ were significantly different from each other. The estimate for one cell $(537 \mathrm{msec})$ is probably inflated; one observer was very slow $(980 \mathrm{msec})$ in the first session for that condition. All other comparisons indicate that same response times were equivalent when the configuration changed, whether or not the same elements repeated.

These results confirm those of Felfoldy (1974). One reason orthogonal tasks are performed more slowly than are univariate tasks is that observers respond more readily when stimuli repeat than when they do not. The results further agree with the suggestion that the stimulus must first be processed before its attributes can be operated upon. Repeating the identical stimulus facilitated performance, repeating the identical elements but with a change of configuration did not reliably facilitate response times.

The stimuli for this experiment were all presented in the same place on the oscilloscope screen. It is possible that repeating the same elements but changing the configuration produced slower response times for another reason. Responses could have been slowed because the elements occurred in slightly different places on successive trials; the lines that formed a rectangle were in a different location than those that formed a number sign. To evaluate this, the experiment was repeated with only two observers and with successive stimuli occurring at random locations about the screen. On any trial a stimulus could be centered anywhere within an $8 \times 8 \mathrm{~cm}$ area. The elements never occurred in the same physical place from trial to trial for any condition.

The form of the data for both observers in this modified condition was identical with that in the reported study; both observers responded fastest when the identical stimulus repeated and there were no regular differences between the other conditions. Although presenting stimuli in a known location might be expected to allow for improved overall performance in identification tasks, it appears that knowing precisely where the stimulus will occur does not account for the object repetition effect found in Experiment 1.

\section{EXPERIMENT 2}

The types of results found in information processing tasks of the sort being discussed here have generally been studied using perceptual patterns as the stimuli. Similar results have also been reported in the classification of words (King, Gruenewald, \& Lockhead, in press). When animal terms were bivariately classified according

Table 1

Mean Response Times (RT) in Milliseconds to Each Sequential Condition Examined in Experiment 1

\begin{tabular}{|c|c|c|c|c|c|c|c|c|}
\hline & \multicolumn{4}{|c|}{ Same Response } & \multicolumn{4}{|c|}{ Different Response } \\
\hline & \multicolumn{4}{|c|}{ Configuration } & \multicolumn{4}{|c|}{ Configuration } \\
\hline & \multicolumn{2}{|c|}{ Repeats } & \multicolumn{2}{|c|}{ Changes } & \multicolumn{2}{|c|}{ Repeats } & \multicolumn{2}{|c|}{ Changes } \\
\hline & \multicolumn{2}{|c|}{ Stimulus } & \multicolumn{2}{|c|}{ Elements } & \multicolumn{2}{|c|}{ Height } & \multicolumn{2}{|c|}{ Height } \\
\hline & Identical & Different & Identical & Different & Same & Different & Same & Different \\
\hline Sequential Stimuli & $\begin{array}{l}1-1,2-2, \\
3-3,4-4, \\
\text { A-A, B-B, } \\
\text { C-C, D-D }\end{array}$ & $\begin{array}{l}1-3,2-4 \\
A-C, B-D\end{array}$ & $\begin{array}{l}\text { 1-A, 2-B, } \\
\text { 3-C, 4-D }\end{array}$ & $\begin{array}{l}\text { 1-C, 2-D } \\
3-A, 4-B\end{array}$ & $\begin{array}{l}1-2,3-4 \\
A-B, C-D\end{array}$ & $\begin{array}{l}1-4,2-3 \\
A-D, B-C\end{array}$ & $\begin{array}{l}1-B, 2-A \\
3-D, 4-C\end{array}$ & $\begin{array}{l}1-D, 2-C \\
3-B, 4-A\end{array}$ \\
\hline RT & 431 & 507 & 502 & 537 & 516 & 508 & 545 & 556 \\
\hline
\end{tabular}

Note-Sequential means both possible orders of events. For example, from the last entry in the second row, Stimulus 4 followed by Stimulus A, or A followed by 4. The stimulus notations indicate the stimuli as shown in Figure 1. 
to the referent's size (dimension X, big or little) and predacity (dimension $Y$, nice or nasty), orthogonal tasks were generally performed more slowly than univariate tasks, and correlated tasks were performed fastest of all. No word repetitions occurred in that study. This result indicates that the slow performance in the orthogonal conditions could not be due to fewer stimulus repetitions in that task compared to the univariate tasks. Yet, the results of Experiment 1 suggest that performance on orthogonal tasks is slow because of the smaller number of stimulus repetitions. If there is only one reason that orthogonal tasks are performed slowly, these conclusions are inconsistent.

The conclusion from Experiment 1 might be incomplete. Perhaps there are sequential effects attributable to the repetition of stimulus attributes (sometimes called partial repetition effects) and these effects are smaller than Experiment 1 could uncover but large enough to produce the outcomes reported by King et al. (in press). Thus, orthogonal tasks may be slow because of relatively few repetitions of objects and of attributes, and perhaps the first study was not sensitive enough to show these effects. Alternatively, consistent failure to find partial repetition effects would call for a reason, in addition to sequential effects due to item repetitions, that orthogonal tasks are sometimes performed more slowly than are univariate tasks.

Experiments 2 and 3 were conducted to evaluate, first, the effects of stimulus repetitions using words and, second, the effects of repeating identical attribute levels with different stimuli. Accordingly, Experiment 2 is a replication of Experiment 1 using words as stimuli. Experiment 3 is a more extensive test than Experiment 2 to determine if there are attribute or feature repetition effects in addition to those due to object repetitions.

\section{Method}

Stimuli. The stimuli were 36 of the 40 words scaled by King et al. (in press). These words and a two-dimensional display of the similarity relations between them are shown in Figure 2. Analogous to Experiment 1, where length of the vertical line constituted one dimension and length of the horizontal line constituted the other dimension, in this study size and predacity take corresponding roles.

Subjects. Four students experienced in perception tasks served as paid subjects.

Procedure. Each observer sat in front of the oscilloscope and two-response keyboard; the left hand was on the left key and the right hand was on the right key. When a word was presented on the screen, the observer pressed the left or right key in response. Classification took place on both the size and predacity dimensions with levels and tasks balanced across subjects with respect to the left and right keys. Two observers sorted according to size and then, on the next day, according to predacity; the other two observers sorted predacity first and then size. Stimuli were randomly selected with replacement for each trial. A stimulus remained on the screen until the response occurred, or until $1 \mathrm{sec}$ elapsed, whichever came first, the screen then erased and the next stimulus appeared in about $520 \mathrm{msec}$. Each subject received 50 practice trials followed by two blocks of 200 trials on each day. There was a brief rest between the two blocks. The second block of 200 responses per subject per task was used in the analy sis.

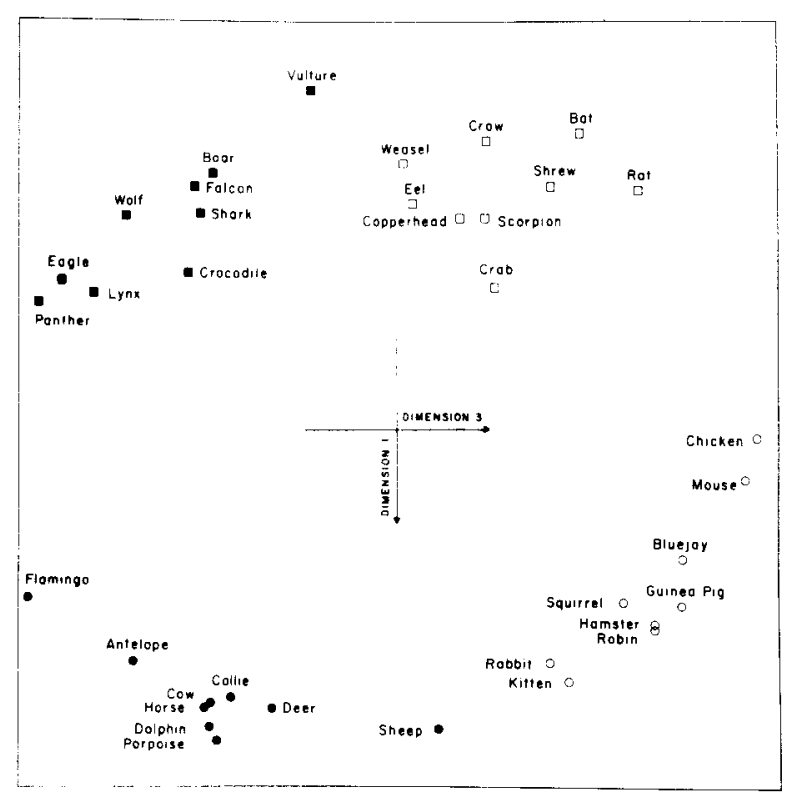

Figure 2. The stimuli used in Experiments 2 and 3 and their similarity relations adapted from King, Gruenewald, and Lockhead (in press). Circles and squares denote terms classified as nice and as nasty, respectively. Filled and open labels denote terms ctassified as big and as little, respectively.

\section{Results}

Of all trials, $3.9 \%$ were errors or trials on which no response occurred. These trials and the next trial were discarded from the analysis. According to a treatments (sequential response times) by subjects analysis of variance, there was no main effect of task $(F<1)$; words were sorted according to size about as fast $(357 \mathrm{msec})$ as they were sorted according to predacity $(366 \mathrm{msec})$. The effect of stimulus sequence was statistically reliable $[F(4,12)=20.8, p<.001]$. The sequential response times are shown in the upper half of Table 2. According to a Newman-Keuls test $(\alpha=.01)$, responses were faster when the identical word repeated than in all other conditions and no other differences were reliable. Only one other condition was reliably $(\alpha=.05)$ different from any other: When the same cell but a different word repeated, sorting times were significantly faster $(355 \mathrm{msec})$ than when a different response was called for and the current word was different from the previous word on just one attribute (396 msec). As an example, when sorting was based on size, responses tended to be faster when "collie" was followed by "antelope" than when "collie" was followed by "hamster."

These results with words as stimuli mirror those of Experiment 1. Sorting time was facilitated when the identical configuration (word) repeated, but no reliable facilitation of the same response was found when the dimensional levels were repeated. Speed differences in sorting tasks depend at least partially on repetitions of identical stimuli but depend relatively little on repetitions of jrrelevant attributes. 
Table 2

Average Response Time to Each Sequential Condition Evaluated in Experiments 2 and 3

\begin{tabular}{|c|c|c|c|c|c|c|c|c|c|c|c|c|c|c|c|}
\hline & & & & & & & & & & \multicolumn{6}{|c|}{ Different Response } \\
\hline & \multicolumn{9}{|c|}{ Same Response } & \multicolumn{6}{|c|}{ Irrelevant Level } \\
\hline & \multicolumn{3}{|c|}{ Repeat the Word } & \multicolumn{3}{|c|}{ Repeat the Cell } & \multicolumn{3}{|c|}{ Different Cells } & \multicolumn{3}{|c|}{ Same } & \multicolumn{3}{|c|}{ Different } \\
\hline & $\mathrm{S}$ & $\mathbf{P}$ & $\mathbf{R}$ & $\mathbf{S}$ & $\mathbf{P}$ & $\mathbf{R}$ & $S$ & $\mathbf{P}$ & $\mathbf{R}$ & $\mathrm{S}$ & $\mathbf{P}$ & $\mathbf{R}$ & $\mathbf{S}$ & $\mathbf{P}$ & $\mathbf{R}$ \\
\hline $\begin{array}{l}\text { Experiment } 2 \\
\text { Experiment } 3\end{array}$ & 321 & 277 & 300 & $\begin{array}{l}346 \\
463\end{array}$ & $\begin{array}{l}364 \\
423\end{array}$ & $\begin{array}{l}355 \\
443\end{array}$ & $\begin{array}{l}374 \\
466\end{array}$ & $\begin{array}{l}390 \\
429\end{array}$ & $\begin{array}{l}382 \\
447\end{array}$ & $\begin{array}{l}383 \\
482\end{array}$ & $\begin{array}{l}410 \\
448\end{array}$ & $\begin{array}{l}396 \\
465\end{array}$ & $\begin{array}{l}359 \\
483\end{array}$ & $\begin{array}{l}387 \\
438 \\
\end{array}$ & $\begin{array}{l}373 \\
461 \\
\end{array}$ \\
\hline
\end{tabular}

Note-S refers to size, $P$ refers to predacity, and $R$ refers to the results evaluated in the reported analyses.

\section{EXPERIMENT 3}

This experiment replicated Experiment 2 while eliminating stimulus repetitions and collecting more data. Repetitions of attribute levels were examined independently of stimulus repetitions. A second goal of the experiment was to determine whether the similarity relations of the sorted words reflect sorting times. Similarity relations have been shown to predict sorting times in earlier semantic sorting studies (King et al., in press).

\section{Method \\ Stimuli. The stimulus words and materials were those used in Experiment 2. \\ Subjects. Twenty-four paid subjects were obtained from the university undergraduate population. \\ Procedure. The procedure here was much like that in Experiment 2. Twelve subjects categorized the randomly presented stimuli according to predacity on Day 1 and according to size on Day 2. The other 12 observers classified by size first and then by predacity. Each of the 48 sessions began by familiarizing the observer with the stimuli and their classification for that task. Then there were 50 practice trials followed by 220 data trials. Stimuli within each session were presented randomly but, unlike in Experiment 2, with the restriction that no word could repeat on successive trials.}

\section{Results}

The average error rate was $3.4 \%$ of the 10,560 responses. Incorrect responses and responses to stimuli following an error were discarded. The average reaction time was calculated for each observer in each task and for each of the four sequential effects (same or different response while at the same level or at the different level of the irrelevant dimension). According to a task (size vs. predacity) by treatments (four sequential conditions) by subjects analysis of variance, words were sorted marginally faster according to predacity than according to size [434 vs. $473 \mathrm{msec} ; \mathrm{F}(1,23)=4.46, \mathrm{p}<.05]$ and the effect of sequence was reliable $[F(3,69)=4.45$, $p<.01]$. The sequential response times are shown in the lower half of Table 2 . It is clear by inspection that the sequential effect is due to whether or not the response changes. When the same response as that on the previous trial was called for, observers were about $19 \mathrm{msec}$ faster than when they had to respond with the other hand. There were no sequential effects due to the repetition of attribute levels in these data. If there are any partial repetition effects that this study could not detect, they must be very small. The experiment was sensitive to a 19-msec difference (changed response), while the numerical estimate of any partial repetition effect is only about $4 \mathrm{msec}$.

The words used in this experiment were 36 of the 40 words used in the classification tasks reported by King et al. (in press). In that study, the similarity relations between the words correlated with response times and with errors. Two measures, called $B$ and $W$, were introduced there. B is the distance in similarity space, as determined by direct distance similarity scaling, between each word in one stimulus cell and all words in the other cell for that task. This measure of the distance between words in the two cells will be large if the two cells are well separated in similarity. For example here, the nice and nasty words appear further separated from each other in similarity space (Figure 2) than are the big and little words. The calculated value of $B$ is larger in the first case (predacity) than in the second case (size), and the words were sorted faster according to predacity than according to size. This is consistent with the negative correlation between $B$ and response times reported earlier (King et al., in press). $W$ is the average distance in similarity space of each word from every other word within each sorted cell. This is a measure of the variability or spread of the items in each cell and will be large if the items to be sorted into the same category are separated in similarity. In the King et al. study, large values of $W$ were associated with slow sorting times. Although $\mathrm{W}$ does not differ very much between conditions in this study, it is somewhat larger for the nasty animals than for the nice animals in the predacity task, and the nasty animals were responded to more slowly $(450 \mathrm{msec})$ than were the nice animals $(432 \mathrm{msec})$ in this condition. There are not enough different conditions to test whether $\mathrm{B}$ and $\mathrm{W}$ are importantly related to response times in this study, but the results here are consistent with the previous suggestion that it is distance in similarity space, and not particular stimulus attributes, that predicts response times.

\section{DISCUSSION}

When the identical object repeats on successive trials, the time required to classify the stimulus 
according to a particular attribute is less than when the attributes repeat on successive trials but in a different configuration. This finding holds for both geometric patterns and animal terms. When a decision has just been made about an attribute of an object, and that object occurs again immediately, the object is recognized as identical to the previous stimulus and the same response is very quickly executed. If, however, an aspect of the object is different on this trial than on the last trial, either in terms of the value of one of the stimulus attributes or in terms of how identical attributes configure, added processing is required. This is the prediction of an earlier model that proposed that a stimulus is initially processed holistically and only then can it be analyzed into its attributes (Lockhead, 1972). Also, these sequential findings are consistent with those of Felfoldy (1974), who additionally showed that the magnitude of the effect of sequential repetitions in reducing decision time decreases as the time between stimulus presentations increases. This fact may reflect the observer's memory for the previous stimulus and that he does not have to analyze the stimulus for an attribute if the object is perceived to have repeated.

There appear to be two classes of repetition effects in the literature relevant to this discussion. In some studies responses are speeded if all attributes of a stimulus repeat and do so in the same configuration (identity), but responses are not speeded if some characteristic of the stimulus, an attribute or the configuration, is changed on successive trials (nonidentity) (Felfody, 1974; Keuss, 1977; the present paper). In other studies response times are facilitated both when there is identity repetition and when only some attributes repeat. The latter results have been labeled as partial repetition effects (PREs) and have been reported in classification tasks (Biederman, 1972, Note 2), identification tasks (Higgins \& Gettys, 1977), attribute detection tasks (Keuss, 1977), when the probability of occurrence of a subject-predicted attribute is manipulated (Geller, 1974), and when stimulus-response compatibility is different on successive trials (Shaffer, 1965). Thus, some studies report that responses are fast when the entire object repeats, but responses are not necessarily facilitated (depending on similarity) when an attribute repeats; other studies report that responses are speeded as long as relevant attributes repeat.

These two classes of findings are consistent with the differences between two classes of stimuli: whether they are integral or not (Lockhead, 1966; Monahan \& Lockhead, 1977). The perceptual and semantic stimuli used in the studies reported here are integral and are appropriate to study the holistic model being pursued. In these studies, there is facilitation of response times when the entire stimulus repeats. If the stimulus is perceived to be different on the current trial, it must be analyzed before the value of an attribute can be judged and, hence, responses are not as fast. Other two-stage models, such that of Bamber (1969), make a similar prediction in this instance (cf. Keuss, 1977).

In contrast, the stimuli used in studies reporting partial repetition effects seem to have not been integral. If so, this would mean that the two- and threedimensional analyzable stimuli in those studies have each been, psychologically, two or three different objects for the observers. The information processing effects of varying multiple objects may be different from the effects of sequential changes of parts of a single object. For example, the stimuli in two of the studies reported by Higgins and Gettys (1977) were schematic faces formed by an oval subtending approximately 9 by $14 \mathrm{deg}$ of visual angle. The oval surrounded two lines (eyes) that could be horizontal or vertical, two lines forming an angle (nose) that could be large or small, and a curved bar (mouth) that could be narrow or wide. The critical dimensions were the nose and mouth in Experiment 2 and the eyes, nose, and mouth in Experiment 3 . The distance between adjacent features was at least $3 \mathrm{deg}$ of visual angle. This separation is sufficient that the entire experimenterdefined stimulus could not be fully seen in a single fixation, and the observers had to look at attributes one at a time; accordingly, the stimuli could not have been integral. Higgins and Gettys quote one observer as saying, "First I saw the eyes, then the nose, then the mouth" (1977, p. 302). The authors followed this observation by analyzing their data in terms of the subjects' reported order of processing and in terms of which attribute was relevant to the task. They found that the size of the partial repetition effect was markedly influenced by the order in which individual observers processed the stimuli. This means that there was opportunity for response facilitation due to object repetition every time there was repetition of any of the experimenters' attributes. Accordingly, a holistic model would predict a repetition effect for each attribute repetition when the composite stimuli are not integral. Moreover, a holistic model would predict that the more dimensions (objects) that repeated between two successive nonintegral stimuli, the greater would be the magnitude of the effect on the eventual overt response. By this analysis, experimental studies of sequential response times with nonintegral stimuli can reveal how observers sample information in a display, but such studies do not seem to address how attributes of a single object are analyzed.

This interpretation may account for other PREs reported in the literature as well. Consider the studies in which Biederman (1972, Note 2) found a PRE using the size and color of a circle and the tilt of an interior diameter as the stimulus dimensions. We now know that size of circle and tilt of diameter are nonintegral dimensions (Garner \& Felfoldy, 1970), as are color and shape (Handel \& Imai, 1972). Accordingly, Biederman's stimuli were at least partly nonintegral and thus might 
be expected to be processed an attribute (or object) at a time, just as were the attributes of Higgins and Getty's (1977) faces and some attributes of Keuss' (1977) geometric patterns. Consider, as an example, the first defined orthogonal task (called filtering) in Table 1 in Biederman's (1972) extensive classification study. There were four possible responses, the middle or index finger of the left or right hand. There were eight possible stimuli, two sizes, two colors, and two line tilts. For the considered example condition, line tilt was irrelevant. When successive stimuli were the same color, successive correct responses called for the same hand (left for green and right for red). Since repeating the identical object facilitates responses, and since these dimensions are nonintegral, repeating the color should produce faster response times than not repeating the color. This outcome shows up as a partial repetition effect when the data are analyzed as if the multidimensional nonintegral stimuli are single objects. The predicted outcome is similar for the other tasks in that study as well, and the results are consistent with a "self-terminating, feature-testing, contingent model of processing" (Biederman, 1972, p. 219). This conclusion applies to how multiple objects in a single display are processed. It does not reveal how attributes of single objects are processed. There seems as yet no empirical basis to predict a partial repetition effect with integral stimuli.

The various results argue that the psychological nature of multidimensional stimuli must be understood if we are to determine a successful model of how attributes are processed. An integral two-dimensional stimulus (one object) is processed differently than is a nonintegral two-dimensional stimulus (two objects), and the interpretation of the data must reflect this fact.

The sequential repetition results reported in this paper concern the processing of integral stimuli and predict that performance in orthogonal tasks is slower than is that in univariate tasks and in correlated tasks. This is because of the different probabilities of repeating the stimulus in these situations. But performance must be even slower in the orthogonal tasks than can be accounted for by stimulus repetitions. This is because the orthogonal tasks are slow even when there are no stimulus repetitions (King et al., in press), and that finding is apparently not due to partial repetition effects (the present Experiment 3). Another factor is needed to account for slow response times in orthogonal vs. univariate or correlated tasks. A possible explanation lies in consideration of the similarity differences between univariate and orthogonal stimulus sets. The distance in similarity space over which stimuli vary is greater in orthogonal tasks than it is in univariate tasks. For example, in Figure 2, an orthogonal task requires observers to decide about words that vary over the full similarity space, while univariate tasks require only half the semantic distance, on the average, between items. The average distance in similarity space between items assigned the same response (W) may be positively correlated with response time (cf. King et al., in press; the present Experiment 2). When attention must span further in similarity space, perhaps the task becomes more difficult (cf. Dixon \& Just, 1978). This interpretation is consistent with findings in psychophysical tasks in which the discrimination between items, in unidimensional absolute-judgment situations, becomes more difficult as the range over which stimuli vary increases (Gravetter \& Lockhead, 1973). Distance in similarity space between loudnesses and line lengths produces effects consistent with the range effect obtained when words are the stimuli; a large range results in poor performance. A stimulus-range effect is a possible explanation for the relative difficulty of orthogonal tasks.

The suggestion that similarity range and discriminability are inversely related predicts, for example, that the orthogonal task reported in Experiment 1, using rectangles and number signs as stimuli, should be performed faster when either rectangles or number signs, but not both, are the stimuli. This is because the range between stimuli in similarity space is likely greater with the eight stimuli than with the four stimuli. The suggestion that responses are facilitated when the identical stimulus, not just the identical elements, repeats, also predicts faster response times with the four stimuli because the identical stimulus would repeat twice as often on successive trials. Accordingly, these data and analyses suggest two factors are important to the classification of integral stimuli: Responses are faster and more accurate if the same object repeats than if it does not, and objects are more readily classified if the similarity distance over which the to-be-classified stimuli range is small than if it is large.

\section{REFERENCE NOTES}

1. Gruenewald, P. J., \& Lockhead, G. R. Sorting perceptual sets. Paper presented at the annual meeting of the Psychonomic Society, Washington, D.C., November 1977.

2. Biederman, 1. Human performance in contingent information-processing tasks. Human Petformance Center Technical Report No. 3, University of Michigan, 1966.

\section{REFERENCES}

BAmbER, D. Reaction times and error rates for "same""different" judgments of multidimensional stimuli. Perception \& Psychophysics, 1969, 6, 169-174.

Bertelson, $P$. Sequential redundancy and speed in a serial two-choice responding task. Quarterly Journal of Experimental Psychology, 1961, 13, 90-102.

Bertelson, P. Serial choice reaction time as a function of response vs. signal-and-response repetition. Nature, 1965, 206, 217-218. 
Biederman, I. Human performance in contingent information-processing tasks. Journal of Experimental Psychology, 1972, 93, 219-238.

Dixon, P., \& JusT, M. A. Normalization of irrevelant dimensions in stimulus comparisons. Journal of Experimental Psychology: Human Perception and Performance, 1978, 4, 36-46.

Felfoldy, G. L. Repetition effects in choice reaction time to multidimensional stinuli. Perception \& Psychophysics, 1974, 15, 453-459.

Fitts, P. M., \& Biederman, I. S-R compatibility and information reduction. Journal of Experimental Psychology, $1965,69,408-412$.

GARNER, W. R. The processing of information and structure. New York: Wiley, 1974.

Garner. W. R., \& Felfoldy, G. L. Integrality of stimulus dimensions in various types of information processing. Cognitive Psychology, 1970, 1, 225-241.

GELLER, E. S. Prediction outcome of a response-irrelevant stimulus as a determinant of choice reaction time. Journal of Experimental Psychology, 1974, 103, 546-552.

Gravetter, F., \& Lockhead, G. R. Criterial range as a frame of reference for stimulus judgment. Psychological Review, 1973, 80, 203-216.

HANDEL, S., \& ImaI, S. The free classification of analyzable and unanalyzable stimuli. Perception \& Psychophysics, $1972,12,109-116$.

Higgins, D. L., \& Getrys, C. F. A partial repetition effect in choice reaction time for multidimensional stimuli. Perception \& Psychophysics, 1977, 21, 298-306.

Hutchinson, W., \& Lockhead, G. R. Similarity as distance: A structural principle for semantic memory. Journal of Experimental Psychology: Human Learning and Memory, $1977,3,660-678$.

KEuss, P. J. G. Processing of geometrical dimensions in a binary classification task: Evidence for a dual process model. Perception \& Psychophysics, 1977, 21, 371-376.

King, M., Gruenewald, P. J., \& Lockhead, G. R. Classifying related stimuli. Joumal of Experimental Psychology: Human Learning and Memory, in press.

LockHEAD, G. R. Effects of dimensional redundancy on visual discrimination. Joumal of Experimental Psychology, 1966, 72, 95-104.

LockheAd, G. R. Processing dimensional stimuli: A note. Psychological Review, 1972, 79, 410-419.

LockHEAD, G. R., \& King, M. C. Classifying integral stimuli. Journal of Experimental Psychology: Human Perception and Performance, 1977, 3, 436-443.

Monahan, J. S., \& Lockhead, G. R. Identification of integral stimuli. Journal of Experimental Psychology: General, 1977, 106, 94-110.

PosNer, M. I. Information reduction in the analysis of sequential tasks. Psychological Review, 1964, 71, 491-504.

PosNer, M. I., \& SNYDER, C. Facilitation and inhibition in the processing of signals. In P. M. A. Rabbitt \& S. Dornic (Eds.), Attention and performance V. Academic Press, 1975. Pp. 669-682.

Selfridge, O. Pandemonium: A paradigm for learning. In Symposium on the mechanization of thought processes. London: HM Stationery Office, 1959.

Shaffer, L. H. Choice reaction with variable S-R mapping Journal of Experimental Psychology, 1965, 70, 284-288.

TAYLOR, B. Dimensional interactions in vibrotactile information processing. Perception \& Psychophysics, 1977, 21, $477-481$.

(Received for publication August 29, 1977; revision accepted April 19, 1978.) 